CASE REPORT

\title{
Guillain Barré syndrome precipitated by the use of antilymphocyte globulin in the treatment of severe aplastic anaemia
}

\author{
B Kaya, C E Davies, H E Oakervee, N C Silver, J Gawler, J D Cavenagh
}

J Clin Pathol 2005;58:994-995. doi: 10.1136/icp.2004.020354

This report describes the case of a 54 year old woman with very severe aplastic anaemia who was treated with antilymphocyte globulin (ALG) and developed Guillain Barré syndrome (GBS). No antecedent infective aetiology was identified. Although there are numerous reports of autoimmune disease after treatment with ALG in aplastic anaemia, and GBS after immunosuppressive treatment, there are none reporting GBS after the use of ALG for severe aplastic anaemia. The occurrence of autoimmune disease after immunosuppressive treatment, in particular ALG, is discussed, together with the possible mechanisms that result from $T$ cell depression.

W e describe a 54 year old woman with severe aplastic anaemia who was treated with antilymphocyte globulin (ALG) and developed Guillain Barré syndrome (GBS). No antecedent infective aetiology was identified. Although there are numerous reports of autoimmune disease after treatment with ALG in aplastic anaemia, and GBS after immunosuppressive treatment, there are none reporting GBS after the use of ALG for severe aplastic anaemia. We will discuss the occurrence of autoimmune disease after immunosuppressive treatment, in particular ALG, and the possible aetiology as a result of $\mathrm{T}$ cell depression.

\section{CASE REPORT}

A 54 year old woman was referred to our hospital with a diagnosis of very severe aplastic anaemia. She had presented two months previously predominantly with severe thrombocytopenia (haemoglobin (Hb), $10.1 \mathrm{~g} /$ litre; white cell count (WCC), $3.2 \times 10^{9}$ /litre; neutrophils, $1.7 \times 10^{9}$ /litre; platelets, $17 \times 10^{9}$ /litre) and had received a course of prednisolone. However, during this period her blood count deteriorated $\left(\mathrm{Hb}, 4.7 \mathrm{~g}\right.$ /litre; WCC, $3.0 \times 10^{9}$ /litre; neutrophils, $0.1 \times 10^{9}$ / litre; platelets, $4 \times 10^{9}$ /litre) and she was referred for further management. Bone marrow examination revealed a hypocellular marrow consistent with a diagnosis of aplastic anaemia with normal cytogenetics. Investigation failed to reveal the underlying aetiology (hepatitis $\mathrm{A}, \mathrm{B}$, and $\mathrm{C}$ viruses, Epstein Barr virus, human immunodeficiency virus, and parvovirus were negative and an autoimmune profile, direct Coombs, sucrose lysis, and Ham's tests were all normal).

Therefore, the patient was given a five day course of equine ALG (lymphoglobulin; Sangstat, Cambridge, Massachusetts, USA) at a dose of $15 \mathrm{mg} / \mathrm{kg} /$ day (10 vials a day) after an initial test dose. During the treatment she developed culture negative fevers, which responded to hydrocortisone, consistent with serum sickness. Sixteen days after completion of ALG treatment and three days after reduction of the hydrocortisone she awakened with lower limb weakness associated with absent reflexes, sensory loss in a stocking distribution, and urinary retention. The motor weakness progressed over the next 24 hours to involve her upper limbs. Magnetic resonance imaging of the spinal cord and computerised tomography of the brain were both unremarkable. Nerve conduction studies showed a generalised sensorimotor polyneuropathy consistent with the recent onset of acquired inflammatory demyelinating polyneuropathy (GBS). Screening for known precipitants of GBS (Campylobacter jejuni, cytomegalovirus, Epstein Barr virus, Mycoplasma pneumoniae, and human immunodeficiency virus infection) was negative. Lumbar puncture for cerebrospinal fluid was not undertaken in view of the thrombocytopenia. Antiganglioside antibodies were negative.

She received a five day course of intravenous immunoglobulin (IVIg) and intensive neurophysiotherapy over several weeks. She was started on cyclosporin and required a short course of granulocyte colony stimulating factor support. A significant improvement was noted within 48 hours of starting IVIg. She was walking with support at four weeks and independently mobile eight weeks after IVIg treatment. Ten months later she has had a partial haematological recovery $\left(\mathrm{Hb}, 9.5 \mathrm{~g} /\right.$ litre; reticulocytes, $3.0 \times 10^{9}$ /litre; WCC, $4.9 \times 10^{9}$ /litre; neutrophils, $2.2 \times 10^{9}$ /itre; platelets, $81 \times$ $10^{9}$ /litre) and is transfusion independent, although she continues to receive cyclosporin.

\section{DISCUSSION}

GBS is an acute demyelinating polyradiculoneuropathy, characterised by progressive, symmetrical weakness and areflexia, and in severe cases with respiratory muscle and autonomic involvement, although bladder involvement is unusual. It is believed to be caused by autoimmune mechanisms that are predominantly $\mathrm{T}$ cell mediated and is a rare but recognised event after autologous and allogeneic bone marrow transplantation, intensive conditioning regimens, and solid organ transplantation. ${ }^{1}$ GBS is also well documented in conditions where immune dysfunction is a feature, notably Hodgkin ${ }^{2}$ and non-Hodgkin lymphoma, ${ }^{3}$ HIV infection, and numerous autoimmune disorders such as chronic active hepatitis, hypothyroidism, sarcoidosis, Wegener's granulomatosis, and ulcerative colitis. It is postulated that in these situations GBS may arise as a result of viral infection/reactivation or as a result of immune dysregulation, in particular iatrogenically suppressed $\mathrm{T}$ cell function in the case of transplant patients.

Immunosuppressive treatment in itself is not thought to be a risk factor for the development of demyelinating

Abbreviations: ALG, antilymphocyte globulin; GBS, Guillain Barré syndrome; $\mathrm{Hb}$, haemoglobin; IVlg, intravenous immunoglobulin; WCC, white cell count 


\section{Take home messages}

- We describe a 54 year old woman with severe aplastic anaemia who was treated with antilymphocyte globulin (ALG) and developed Guillain Barré syndrome (GBS)

- This is the first report of GBS after the use of ALG for severe aplastic anaemia

- GBS is thought to be autoimmune in origin, and in this case was probably caused by a reduction in cytotoxic $T$ cell responses that would normally restrict $B$ cell activation as a result of the administration of ALG

neuropathies. Supporting data for this include reports of GBS developing as patients were weaned off steroids and the fact that GBS is also successfully treated by immunosuppressive treatments, such as cyclosporin A. Steroids can also be beneficial in the management of chronic inflammatory demyelinating polyneuropathy. However, there are instances where GBS developed after other immunosuppressive regimens. FK506 treatment in liver transplant recipients, ${ }^{4}$ cyclosporin A in renal transplant recipients, ${ }^{5}$ and cyclosporin A in rodent experimental studies resulted in demyelinating neuropathies. ${ }^{6}$ Other immunosuppressive agents, such as Campath- $1 \mathrm{H}$, are also reported to induce autoimmune conditions-for example, a high incidence of Graves' disease was reported after the use of Campath-1H in patients with multiple sclerosis. ${ }^{7}$ In some of these instances, an immune mechanism was suggested because symptomatic improvement was seen after plasma exchange or IVIg.

\section{"Immunosuppressive treatment in itself is not thought to be a risk factor for the development of demyelinating neuropathies"}

Aplastic anaemia is thought to be mediated by a cellular immune reaction directed against haemopoetic stem or progenitor cells. ALG derived from horse or rabbit serum appears to act by reducing cytotoxic $\mathrm{T}$ cells and by releasing haemopoetic growth factors from certain $\mathrm{T}$ cells, thereby dampening down the cellular immune response. ${ }^{8}$ Reduced cytotoxic T cell responses that would normally restrict B cell activation but allow growth of haemopoetic progenitor cells may increase the likelihood of autoimmune disorders arising. Although there are no reports of GBS after treatment with
ALG for aplastic anaemia, there are reports of other autoimmune disorders after ALG. Autoimmune thyroid disease, although rare, is described most frequently, ${ }^{9}$ and autoimmune haemolytic anaemia ${ }^{10}$ and fibrosing alveolitis have also been reported. ${ }^{11}$ In addition, pre-existing multiple sclerosis and fibrosing alveolitis were exacerbated after ALG, suggesting the potential of ALG to trigger and perpetuate autoimmune disease.

\section{Authors' affiliations}

B Kaya, C E Davies, H E Oakervee, J D Cavenagh, Department of Haematology, Barts and the London NHS Trust, London EClA 7BE, UK N C Silver, J Gawler, Department of Neurology, Barts and the London NHS Trust

The patient gave her informed consent for this case report to be published.

Correspondence to: Dr J D Cavenagh, Department of Haematology, St Bartholomew's Hospital, West Smithfield, London EClA 7BE, UK; j.d.cavenagh@qmul.ac.uk

Accepted for publication 20 January 2005

\section{REFERENCES}

1 Bulsara KR, Baron PW, Tuttle-Newhall JE, et al. Guillain Barré syndrome in organ and bone marrow transplant patients. Transplantation 2001;71:1169-72.

2 Lisak RP, Mitchell M, Zweiman B, et al. Guillain Barré syndrome and Hodgkin's disease: three cases with immunological studies. Ann Neurol 1977;1:72-8

$3 \operatorname{Re} D$, Schwenk A, Hegener $P$, et al. Guillain Barré syndrome in a patient with non Hodgkin's lymphoma. Ann Oncol 2000;11:217-20.

4 Wilson JR, Conwit RA, Eidelman BH, et al. Sensorimotor neuropathy resembling CIDP in patients receiving FK506. Muscle Nerve 1994;17:528.

5 Palmer BF, Toto RD. Severe neurological toxicity induced by cyclosporin A in three renal transplant patients. Am J Kidney Dis 1991;18:116-21.

6 McCombe PA, Van der Krecke, Pender MP. The effects of prophylactic cyclosporin A on experimental allergic neuritis (EAN) in the Lewis rat. Induction of relapsing EAN using low dose cyclosporine. Am J Neuroimmunol 1990;28:131-40.

7 Coles AJ, Wing M, Smith S, et al. Pulsed monoclonal antibody treatment and autoimmune thyroid disease in multiple sclerosis. Lancet 1999;354:1691-5.

8 Termura M, Kobayashi S, Iwabe K, et al. Mechanism of action of antithymocyte globulin in the treatment of aplastic anaemia: in vitro evidence for the presence of immunosuppressive mechanism. Br J Haematol 1997;96:80-4.

9 Todd A, Todd J. Graves' disease following successful treatment of severe aplastic anaemia with antilymphocyte globulin. Clin Lab Haematol 1999;21:69-70.

10 Prchal JT, Huang ST, Court WS, et al. Immune haemolytic anaemia following administration of ATG. Am J Haematol 1985; 19:95-8.

11 Zomas A, Marsh JCW, Harrison NK, et al. Rapid progression of fibrosing alveolitis and thyrotoxicosis after antithymocyte globulin therapy for aplastic anaemia. Ann Haematol 1995;71:49-51. 